\title{
THE ALLOCATION OF INTANGIBLE RESOURCES: THE ANALYTIC HIERARCHY PROCESS AND LINEAR PROGRAMMING
}

\author{
Thomas L. Saaty \\ Luis G. Vargas \\ University of Pittsburgh \\ Pittsburgh, PA 15260 . USA \\ saaty@katz.pitt.edu / vargas@katz.pitt.edu
}

\begin{abstract}
Allocating resources with linear programming (LP) is based on using absolute measurements from existing scales for the coefficients and variables in the objective function and in the constraints. An existing scale is one which has been developed through long usage or experimentation (the foot, the angstrom); such scales always involve a unit that is applied to measure the objects in the set. We show in this paper that linear programming problems can also be formulated using relative measurement. Linear programming formulations using relative measurements can then be applied to optimization problems involving a mix of tangibles with existing scales, tangibles with no scales, and intangibles. We prove that the two types of problem; LP with measurements from known scales and relative LP, yield solutions that are equivalent to within a multiplicative constant and illustrate with a simple example how to deal with intangible coefficients and variables.
\end{abstract}

\section{Introduction}

We have two conceptually challenging and difficult problems to discuss in this note: (1) How to reduce any linear programming model to a model that uses only relative ratio scales, and (2) What to do about allocating resources to social, psychological and political phenomena.

In a typical linear programming (LP) problem, the variables are all measured on the same scale and to formulate an LP model one must have an existing physical scale on which to measure the variables. The constraints in LP are prescribed in terms of amounts of different types of resources. All the coefficients in an inequality constraint belong to the same scale, but these coefficients may differ from constraint to constraint: money, weight, volume, time and other resources. The coefficients in the objective function are measured in yet a different scale, typically something to do with money such as cost or profit that one needs to minimize or maximize.

In linear programming measurements must belong to a ratio scale (see Roberts, 1991) and all the variables must be measured with the same unit. A ratio scale is a mapping from the set of elements being measured to a numerical space that is invariant to within a similarity transformation, i.e., any other scale measuring the same property on the same set of objects will differ from the first scale by a multiplicative constant. Thus, ratio scale measurements of a finite set of objects can be standardized (i.e., normalized to unity) by dividing them by the sum of all the elements. It is because of this invariance to similarity transformations that LP can be used with intangibles of which there is a near infinite number and variety and for each of which there is little hope for creating and disseminating for wide use a separate measurement scale.

Relative scale values for a set of objects can be obtained by simply normalizing the measures obtained for them on an absolute scale. Or, one can construct a ratio of the absolute numbers for each pair and form the AHP pairwise comparison matrix for the entire set of objects (Saaty 1994, 1996). One then derives a relative scale of values by computing the principal eigenvector of the matrix, whose coefficients are the same as those obtained by using the simple normalization process. The AHP process is unnecessary if one has a preexisting scale and a unit (and a physical device) to measure with, but it is invaluable if there is no preexisting absolute scale and one needs to estimate the ratios.

While it is true that one can create a scale of relative measurement by normalizing actual readings from an existing scale, it is also true that relative measurement is a much deeper and more general process in which a scale of relative values is derived from paired comparisons of elements with respect to a shared attribute without the need for a unit of measurement, as it is done in the Analytic Hierarchy Process 
(AHP). We repeat that when actual measurements are used for the comparisons, the AHP gives back that scale as a derived scale normalized by dividing by the sum of all the readings involved.

\section{The LP Model in Priority Space}

We now show that a linear programming model can be represented with relative measurement and that when measurement scales exist, the relative linear programming (RLP) model (with measurements prescribed in relative terms), and the absolute linear programming (LP) model (the usual model with measurements on physical scales) are equivalent. Thus, it should not be construed as unreasonable to construct LP models using solely relative measurement to optimize the allocation of intangible resources.

The Decision Variables: In general one can think of inputs to a decision or actions that are not yet measurable in terms of an agreed upon scale. Examples are, the amount of love and caring one puts into writing a paper, building an addition to a house or garage, the time spent with one's children by doing something together, and the amount of thinking and careful planning for investments. We denote activities or decision variables, in general, by $x_{i}, i=1,2, \ldots, n$, keeping in mind that they may or may not be measurable today. Let $\bar{x}=\left(x_{1}, x_{2}, \cdots, x_{n}\right)^{T}$ be the vector of activities.

The Objective Function: Traditionally LP is used to maximize or minimize some measure of worth. Since it is possible that the objective function may not be expressed in traditional measurements, we first prioritize the decision variables as to their contribution to the objective. What we obtain is a vector of coefficients ${ }_{R} \bar{c}=\left({ }_{R} c_{1},{ }_{R} c_{2}, \cdots,{ }_{R} c_{n}\right)^{T}$ with $\sum_{i=1}^{n}{ }_{R} c_{i}=1$. These coefficients belong to a ratio scale. The objective function is given by: $\operatorname{Max}(\operatorname{Min}){ }_{R} c^{T} \bar{x}$.

The Constraints: The constraints consist of two parts: the left hand side in which the decision variables are used to determine the amount of resources or how the decision variables contribute to the constraints of the problem; and the right hand side coefficients which usually refer to the amount of resources or some level which we may not want the solution to exceed or fall below. Let ${ }_{R} a_{i j}$ be the relative contribution of the $j$ th decision variable to the $i$ th constraint, with $\left.\sum_{j=1}^{n}\right|_{R} a_{i j} \mid=1$ for each $i$. Let ${ }_{R} \bar{b}=\left({ }_{R} b_{1},{ }_{R} b_{2}, \cdots,{ }_{R} b_{m}\right)^{T}$ be the vector of relative worth of the corresponding constraints. We have $\left.\left.\right|_{R} \bar{b}\right|^{T} \bar{e}=1$, where $\bar{e}=(1, \ldots, 1)^{T}$. Let ${ }_{R} A=\left({ }_{R} a_{i j}\right)$ be the matrix of coefficients, where $\left.\right|_{R} A \mid \bar{e}=1$. The constraints are given by: ${ }_{R} \overline{A x} \leq_{R} \bar{b}$.

The optimization problem can now be represented as follows:

$$
\begin{gathered}
\operatorname{Max} \text { (or Min) }{ }_{R} \vec{c}^{T} \bar{x} \\
\text { s.t. }{ }_{R} \overline{A \bar{x}} \leq(\geq)_{R} \bar{b} \\
\bar{x} \geq 0
\end{gathered}
$$

We now show how any linear programming problem can be reduced to this form. The equivalence of two LP solutions implies that they are the same to within a multiplicative constant.

\section{THEOREM: RLP and LP models are equivalent.}

Proof: Consider the standard linear programming problem:

$$
\begin{array}{r}
\operatorname{Max} \sum_{j} c_{j} x_{j} \\
\text { s.t.: } \sum_{j} a_{i j} x_{j} \leq b_{i} . \\
x_{j} \geq 0
\end{array}
$$

To represent this problem in priority space (the space of relative measurement normalized to unity), first normalize to unity the coefficients of the objective function; and then the rows of the matrix of 
coefficients, $A=\left\{a_{i j}\right\}$. In this normalization process we maintain the structure of the problem by adjusting the right-hand side coefficients accordingly. We have:

$$
\begin{gathered}
\operatorname{Max} \sum_{j} \frac{c_{j}}{\sum_{k}\left|c_{k}\right|} x_{j} \\
\text { s.t.: } \sum_{j} \frac{a_{i j}}{\sum_{k}\left|a_{l k}\right|} x_{j} \leq \frac{b_{i}}{\sum_{k}\left|a_{i k}\right|} \\
x_{j} \geq 0
\end{gathered}
$$

Finally, the resulting right-hand side coefficients are normalized to unity leading to a transformation of variables. The problem now has the familiar form:

$$
\begin{array}{r}
\operatorname{Max} \sum_{j}{ }_{R} c_{j} w_{j} \\
\text { s.t.: } \sum_{j} a_{i j} w_{j} \leq_{R} b_{i} \\
w_{j} \geq 0
\end{array}
$$

where ${ }_{R} c_{j}=\frac{c_{j}}{\sum_{k}\left|c_{k}\right|},{ }_{R} a_{i j}=\frac{a_{i j}}{\sum_{k}\left|a_{i k}\right|},{ }_{R} b_{i}=\frac{\frac{b_{i}}{\sum_{k}\left|a_{k k}\right|}}{\sum_{h} \frac{\left|b_{h}\right|}{\sum_{k}\left|a_{h k}\right|}}$ and $w_{j}=\frac{x_{j}}{\sum_{h} \frac{\left|b_{h}\right|}{\sum_{k}\left|a_{h k}\right|}}$.

It should now be clear that the normalized solutions of the two problems are identical, and the $w$ 's and the $x$ 's differ by the multiplicative constant $\sum_{h} \frac{b_{h}}{\sum_{k} a_{h k}}$, and thus belong to the same ratio scale.

COROLLARY: A necessary and sufficient condition for a linear programming model to yield a "valid" solution is that all its measurements belong to ratio scales.

It can be easily shown that the RLP problem corresponding to the dual of the original LP problem given by:

$$
\begin{aligned}
& \operatorname{Min} \sum_{i} b_{l} y_{i} \\
& \operatorname{Min} \sum_{i}{ }_{R} b_{i} v_{i} \\
& \text { s.t.: } \sum_{i} a_{i j} y_{i} \geq c_{j} \text { is the dual of the RLP problem and given by: s.t.: } \sum_{i} a_{i j} v_{i} \geq_{R} c_{j} \text {. } \\
& y_{i} \geq 0 \\
& v_{j} \geq 0
\end{aligned}
$$

One needs to be careful when constructing the RLP problem of the dual because the units of the coefficients in different rows of the LP problem do not belong to the same scale, i.e., all the $a_{i j}, j=1,2, \ldots, n$ are measured in the same unit while the $a_{i j}, i=1,2, \ldots, m$ are not. This dual problem can also be transformed into an equivalent problem in priority space, but the transformation would be the same as in the proof of the theorem.

\section{An Example}

Consider a conflict situation requiring $m$ possible types of effort to resolve it. Assume that there are $\mathrm{N}$ parties involved and that each party has some objectives to fulfill $\left(O_{i j}, j=1,2, \ldots, n_{i}\right)$. Let $x_{i j k}, k=1,2, \ldots, m$, be the amount of effort $\mathrm{k}$ used to attain objective $\mathrm{j}$ of actor $\mathrm{i}$. Let $a_{i j k}$ be the relative amount of effort $\mathrm{k}$ required by the objectives $\left(O_{i j}, j=1,2, \ldots, n_{i}\right)$ of actor $\mathrm{i}$, and $\sum_{j=1}^{n_{1}} a_{i j k}=1, i=1,2, \ldots, \mathrm{N}$.

Let $b_{i k}$ be the relative importance of effort $\mathrm{k}$ to actor $\mathrm{i}$. Thus, $\sum_{j=1}^{n_{1}} a_{i j k} x_{i j k}$ gives the total amount of effort $\mathrm{k}$ used to attain the objectives of actor $\mathrm{i}$. Let $c_{i j k}$ be relative contribution of the use of one unit of effort $\mathrm{k}$ 
to attain objective j. Thus, $\sum_{i=1}^{N} \sum_{j=1}^{n_{i}} \sum_{k=1}^{m} c_{i j k} x_{i j k}$ gives the total contribution of the efforts to attain the objectives of all actors. The problem is to maximize the contribution of the efforts in the attainment of the objectives subject to the availability of the efforts or resources. We have:

$$
\begin{aligned}
& \text { Maximize }: \sum_{i=1}^{N} \sum_{j=1}^{n_{i}} \sum_{k=1}^{m} c_{i j k} x_{i j k} \\
& \text { s.t., } \sum_{j=1}^{n_{l}} a_{i j k} x_{i j k} \leq b_{i k} i=1,2, \ldots, N ; k=1,2, \ldots, m . \\
& x_{i j k} \geq 0, i=1,2, \ldots, N ; j=1,2, \ldots, n_{i} ; k=1,2, \ldots, m .
\end{aligned}
$$

Assume that $\mathrm{N}=2$, and that each party has two objectives, and the resources available are of two types, i.e., $\mathrm{m}=2$. Two parties need to determine how to use two types of resources (diplomacy and military action) to resolve a conflict between them. First, the parties pairwise compare the relative contribution of the resources to attain the objectives. Party 1 thinks that diplomacy contributes slightly more (2) to objective O12 than to objective O11, and Party 2 thinks that diplomacy contributes extremely (9) more to objective $\mathrm{O} 21$ than to objective $\mathrm{O} 22$. Both parties think that military action contributes equally (1) to both of their objectives. Table 4 gives the judgments and priorities.

Table 4. Relative Contribution of Resources to Objectives

Diplomacy
\begin{tabular}{|l|c|c|r|}
\hline Party 1 & 011 & 012 & Priorities \\
\hline 011 & 1 & $1 / 2$ & 0.3333 \\
\hline 012 & 2 & 1 & 0.6667 \\
\hline
\end{tabular}

\section{Military Action}

\begin{tabular}{|l|c|c|c|}
\hline Party 1 & 011 & 012 & Priorities \\
\hline 011 & 1 & 1 & 0.5 \\
\hline 012 & 1 & 1 & 0.5 \\
\hline
\end{tabular}

Diplomacy
\begin{tabular}{|l|c|c|c|}
\hline Party 2 & 021 & 022 & Priorities \\
\hline 021 & 1 & 9 & 0.9 \\
\hline 022 & $1 / 9$ & 1 & 0.1 \\
\hline
\end{tabular}

Military Action

\begin{tabular}{|l|c|c|c|}
\hline Party 2 & 021 & 022 & Priorities \\
\hline 021 & 1 & 1 & 0.5 \\
\cline { 2 - 4 } 022 & 1 & 1 & 0.5 \\
\hline
\end{tabular}

\begin{tabular}{|c|c|c|c|c|c|}
\hline & & 011 & 012 & $\mathrm{O} 21$ & $\mathrm{O} 22$ \\
\hline \multirow[t]{2}{*}{ Party 1} & Diplomacy & 0.33 & 0.67 & $\overline{0}$ & 0 \\
\hline & Mil.Action & 0.5 & 0.5 & 0 & 0 \\
\hline \multirow[t]{2}{*}{ Party 2} & Diplomacy & 0 & 0 & 0.9 & 0.1 \\
\hline & Mil.Action & 0 & 0 & 0.5 & 0.5 \\
\hline
\end{tabular}

The priorities from these comparisons are used to form the matrix of coefficients ${ }_{R} A$ given in Table 5 .

Table 5. Matrix ${ }_{R} A$ of relative use of resources to attain objectives

Thus, for Party 1, the relative use of diplomacy to attain its objectives is 0.3333 and 0.6667 , respectively whereas for Party 2 , they are 0.9 and 0.1 . Both parties may decide that both objectives can call for equal use of military resources, thus the priorities from Table 4 are $(0.5,0.5)$.

Similarly, the parties prioritize the relative benefit of using the resources. Party 1 thinks that diplomacy provides slightly more (2) benefits than military action while Party 2 thinks that military action yields slightly more (2) benefits than diplomacy. Table 6 gives the paired comparisons for both parties.

Table 6. Objective function coefficients, $R \bar{c}$.

Benefit to Party 1 (Party 2)

\begin{tabular}{|l|c|c|c|}
\hline & Diplomacy & Mil.Action & Priorities \\
\hline Diplomacy & 1 & $2(1 / 2)$ & $0.67(0.33)$ \\
\cline { 2 - 4 } Mil.Action & $1 / 2(2)$ & 1 & $0.33(0.67)$ \\
\hline
\end{tabular}

Thus, the objective function is given by:

$$
0.67\left(x_{111}+x_{121}\right)+0.33\left(x_{112}+x_{122}\right)+0.33\left(x_{211}+x_{221}\right)+0.67\left(x_{212}+x_{222}\right) \text {. }
$$

Now the parties must assess the relative importance of the resources. Party 1 prefers diplomacy slightly more (2) than military action, and Party 2 prefers military action strongly more (5) than diplomacy. Table 
7 below summarizes the parties preferences. In addition, the parties are considered equally powerful and hence, their relative preference of the resources are weighted by 0.5 for each of them to obtain the vector of right hand-side coefficients, $R \bar{b}$.

We see that the relative importance of the resources to Party 1 are $0.67 \times 0.5=0.33$ (diplomacy) and $0.33 \times 0.5=0.17$ (mil. action), and $0.17 \times 0.5=0.085$ (diplomacy) and $0.83 \times 0.5=0.415$ (mil. action) to Party 2. The constraints for Party 1 and Party 2 are respectively:

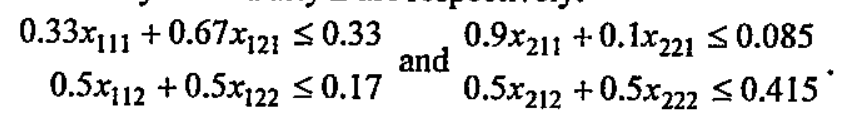

Table 7. RHS Coefficients ${ }_{R} \bar{b}$. Relative importance of the resources

\begin{tabular}{|l|c|c|r|r|}
\hline Party 1 & Diplomacy & Mil.Action & Priorities & RHS Coeff. \\
\hline Diplomacy & 1 & 2 & 0.67 & 0.33 \\
\cline { 2 - 5 } Mil.Action & $1 / 2$ & 1 & 0.33 & 0.17 \\
\hline
\end{tabular}

\begin{tabular}{|l|c|c|r|r|}
\hline Party 2 & Diplomacy & Mil.Action & Priorities & RHS Coeff. \\
\hline Diplomacy & 1 & $1 / 5$ & 0.17 & 0.085 \\
\cline { 2 - 5 } Mil.Action & 5 & 1 & 0.83 & 0.415 \\
\hline
\end{tabular}

The LP problem in priority space is given by:

$$
\begin{aligned}
& \operatorname{Max}: 0.67\left(x_{111}+x_{121}\right)+0.33\left(x_{112}+x_{122}\right)+0.33\left(x_{211}+x_{221}\right)+0.67\left(x_{212}+x_{222}\right) \\
& \text { s.t.; } 0.33 x_{111}+0.67 x_{121} \leq 0.33 \\
& 0.5 x_{112}+0.5 x_{122} \leq 0.17 \\
& 0.9 x_{211}+0.1 x_{221} \leq 0.085 \\
& 0.5 x_{212}+0.5 x_{222} \leq 0.415 \\
& x_{i j k} \geq 0
\end{aligned}
$$

and its solution in normalized form is:

$$
x_{111}=0.33, x_{121}=0, x_{112}=0.08, x_{122}=0.04 ; x_{211}=0, x_{221}=0.28, x_{212}=0.14, x_{222}=0.14 \text {. }
$$

Normalizing the solution for each party we see that Party 1 would use primarily diplomacy (75\%):

$$
x_{111}=0.75, x_{121}=0, x_{112}=0.17, x_{122}=0.08
$$

and Party 2 would use a mix of military action (50\%) and diplomatic effort (50\%):

$$
x_{211}=0, x_{221}=0.50, x_{232}=0.25, x_{222}=0.25 \text {. }
$$

On the other hand, if Party 2 were to change its view of the importance of the resources from $(0.17,0.83)$ to $(0.5,0.5)$, then the new LP problem is given by:

$$
\begin{aligned}
& \operatorname{Max}: 0.67\left(x_{111}+x_{121}\right)+0.33\left(x_{112}+x_{122}\right)+0.33\left(x_{211}+x_{221}\right)+0.67\left(x_{212}+x_{222}\right) \\
& \text { s.t. } 0.33 x_{111}+0.67 x_{121} \leq 0.33 \\
& 0.5 x_{112}+0.5 x_{122} \leq 0.17 \\
& 0.9 x_{211}+0.1 x_{221} \leq 0.25 \\
& 0.5 x_{212}+0.5 x_{222} \leq 0.25 \\
& x_{i j k} \geq 0
\end{aligned}
$$

and its solution now is given by:

$$
x_{111}=0.230, x_{121}=0, x_{112}=0.054, x_{122}=0.025 ; x_{211}=0, x_{221}=0.576, x_{212}=0.058, x_{222}=0.058
$$

which, when normalized for each Party, yields:

$$
x_{111}=0.75, x_{121}=0, x_{112}=0.17, x_{122}=0.08 ; x_{211}=0, x_{221}=0.833, x_{212}=0.083, x_{222}=0.083
$$

This solution can be interpreted as both parties being more prone to use diplomatic effort (75\% for Party 1 and $83 \%$ for Party 2) to resolve the conflict.

In this example, the LP problem was solved by splitting it into two LP problems, one for each party. However, had there been a.resource that is shared by the two parties, the problem would not be solved by doing each problem separately. Assume that diplomatic effort is shared by both parties by combining the 
first and the third constraints above and that both parties have the same power and hence, the coefficients of the new constraint are obtained by multiplying each of the original ones by 0.5 . The right-hand side coefficient of the new constraint is obtained by adding the previous right-hand side coefficients. We have:

$$
\begin{aligned}
& \text { Max : } 0.67\left(x_{111}+x_{121}\right)+0.33\left(x_{112}+x_{122}\right)+0.33\left(x_{211}+x_{221}\right)+0.67\left(x_{212}+x_{222}\right) \\
& \text { s.t., } 0.17 x_{111}+0.33 x_{121}+0.45 x_{211}+0.05 x_{221} \leq 0.58 \\
& 0.5 x_{112}+0.5 x_{122} \leq 0.17 \\
& 0.5 x_{212}+0.5 x_{222} \leq 0.25 \\
& x_{i j k} \geq 0
\end{aligned}
$$

and its solution in normalized form is given by:

$$
x_{111}=0, x_{121}=0, x_{112}=0.019, x_{122}=0.009 ; x_{211}=0, x_{221}=0.932, x_{212}=0.020, x_{222}=0.020
$$

which could be interpreted as Party 2 providing the majority of the diplomatic effort. On the other hand, if the perceived priorities of Party 2 for the use of diplomatic effort shifts from $(0.9,0.1)$ to $(0.33,0.67)$, then the solution shifts drastically to Party 1 to provide the major part of the diplomatic effort. We see this by solving:

$$
\begin{aligned}
\operatorname{Max}: 0.67\left(x_{111}+x_{121}\right)+0.33\left(x_{112}+x_{122}\right)+0.33\left(x_{211}+x_{221}\right)+0.67\left(x_{212}+x_{222}\right) & \\
\text { s.t., } 0.17 x_{111}+0.33 x_{121}+0.17 x_{211}+0.67 x_{221} & \leq 0.58 \\
0.5 x_{112}+0.5 x_{122} & \leq 0.17 \\
0.5 x_{212}+0.5 x_{222} & \leq 0.25 \\
x_{i j k} & \geq 0
\end{aligned}
$$

which yields: $x_{111}=0.800, x_{121}=0, x_{112}=0.055, x_{122}=0.025 ; x_{211}=0, x_{221}=0, x_{212}=0.060, x_{222}=0.060$.

\section{Conclusion}

There are numerous things for which we do not yet have concrete measures. They include the social and political acts of people in which they invest much thought and energy. What should we adopt for a unit act so that we can estimate all other acts as multiples of that unit? We can prioritize some basic acts and select one that is the most typical and use it as a basis for comparing other acts and obtaining values for them. We would then have something that we can measure. The example given in Section 3 shows how relative measurement in an optimization model could be used to suggest actions in a situation where scales are not available. Advancing this way of thinking can have significant practical implications on how we use the other part of our nature, the less physical and more elusive personal, social and political.

\section{References}

Roberts, F.S., "Limitations on Conclusions Using Scales of Measurement," Rutcor Research Report \#4891, Rutgers University, New Brunswick, NJ 08903-5062, 1991.

Saaty, T.L., "Fundamentals of Decision Making and Priority Theory," Vol. VI of the AHP Series, RWS Publications, 4922 Ellsworth Avenue, Pittsburgh, PA 15213, 1994.

Saaty, T.L., "The Analytic Hierarchy Process, Vol. 1 of the AHP Series, RWS Publications, 4922 Ellsworth Avenue, Pittsburgh, PA 15213, 1996 edition.

Saaty, T.L. and K. Peniwati, "The Analytic Hierarchy Process and Linear Programming in Human Resource Allocation," Proceedings of the Fourth International Symposium on the Analytic Hierarchy Process, Simon Fraser University, Burnaby, B:C., Canada, July 12-15, 1996, pp. 492-504.

Stigler, G.J., "The Cost of Subsistence," Journal of Farm Economics 27, 2 (1945) 303-314. 